\title{
Automatic Tire Pressure Controlling and Self Inflating System: A Review
}

\author{
A.V.Wadmare, P.S.Pandure \\ (Department Of Mechanical Engineering, MES College Of Engineering, S.P. Pune Unuversity-411001, India)
}

\begin{abstract}
Tire is the most essential part of automobile and it plays crucial role in ensuring safe driving. Even then, almost every automobile on the road run with either one or more under inflated tires. Detailed survey has come with result that drop in tire pressure by just few psi leads to the reduction in gas mileage, tire life, safe driving and vehicle performance. Unawareness of exact pressure requirement, sudden environmental changes are also some of causes for tire running with improper pressure. Automatic tire pressure controlling and selfinflating system ensures correct pressure in the tire all the time. Drop in pressure is detected by hissing sound made by tire and system will starts refilling the tire automatically according to the requirement of the tire. This system is named automatic because it checks the tire pressure constantly using pressure gauge and accordingly gives alert signals to the driver. Aim of this project is to stabilize all automobile tires with ideal pressure, make system absolutely automatic, achieve satisfactory fuel efficiency, construct an affordable system, increase tire life and reduce accident rate has been achieved by installing the system in vehicle.
\end{abstract}

Keywords: automatic control, safe driving, self-inflating system, tire pressure, vehicle

\section{INTRODUCTION}

A variety of tire monitoring strategies have been proposed to aware driver of low tire pressure. Under inflated tires run on the road due to unawareness of the fact that properly inflated tires can safe tire life up to $20 \%$ which is nine months more of its life span. It can also save fuel from $4 \%$ to $10 \%$, increase braking efficiency up to $20 \%$, and ease the self-steer. The research finding shows that the air pressure in the car drops 10 to $20 \mathrm{kpa}$ a month which is equivalent of adding a $70 \mathrm{~kg}$ person into the car. Inflating accurate tire pressure save the tire from extra heating, explosion and also help decrease maintenance cost, as shown in Fig. 1 [1].

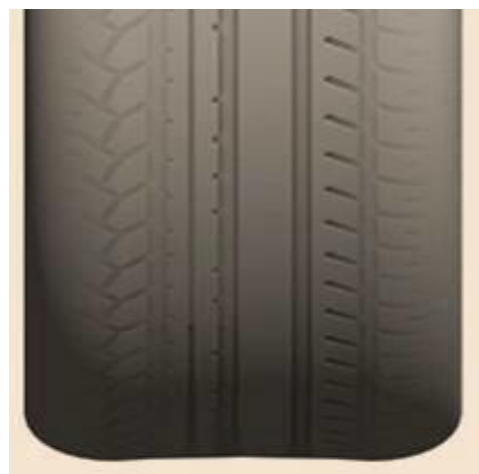

Under-inflation

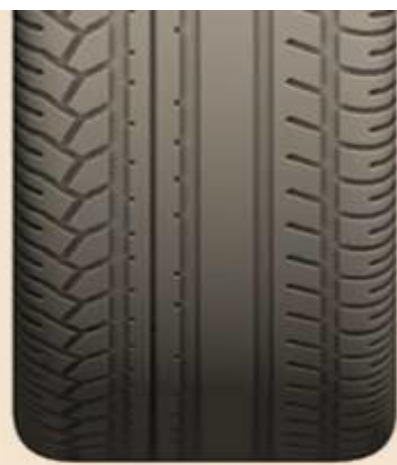

Correct

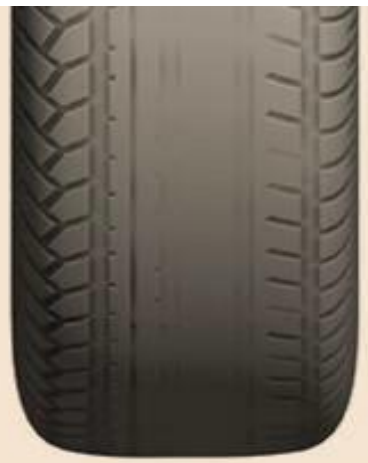

Over-inflation

Fig.1. Tire wear patters observed for different tire inflation pressures [1].

Compressor is used in this system to collect the air from atmosphere, compress it and deliver to the tire for inflation. Under inflated tires tend to wear at the edges more than at the center as the pressure is not sufficient at the center to bear the load. As against this for over inflated tires wear is higher at the center due to bulging of tires. Wearing of the threads causes the skidding of the tire hence leads to major danger accidents. All of related studies shows that under inflation from axle tires result in under steer tendencies while rear axle under inflation creates over steer behavior hence disturbing the car handling. This system is addressed to be automatic as it automatically checks the tire pressure by using pressure gauge and if tire pressure is decreased below ideal condition than the compressor starts to supply the air to refill the tire. It also predicts about the puncture when there is continuous reduction of its set optimal value. The advantage of the system is that it does not require any special attention from user side once after the system being installed. It discards the requirement of checking tire pressure manually, thus saving time and labor. With the recent oil price hikes and ever increasing environmental 
issues, the system addresses a potential development in a gas mileage, tire wear reduction, and an improvement in handling and tire performance in adverse condition. This kind of systems are all- ready being installed in military vehicles and commercial cars for safety purpose and to feel the luxury driving but this system is being introduced for all types of customer vehicles with the absolute motive to give safety assurance and comfort driving. Now with the installation of this system one can drive vehicle under all worst sudden varying environmental conditions like heavy rainfall, snowfall, deserts. Specially at remote places this kind of system proves to be most helpful as repairing devices for maintenance of the automobile are very critically available.At some crucial times like war conditions or any flood condition there is no time to refill the tire with air hence Automatic tire controlling and self- inflating tire system is very essential to be encouraged to install in every automobile to face all tire related issues and enjoy safe and comfort driving [5].

\section{DESIGN METHODOLOGY AND RELATED WORKING}

I. Burase et al. [1] surveyed on automobile air inflating system as shown in the Fig. 2. In this research paper, a new approach is encouraged for automobile a self inflating tire that guarantees that tires will be properly inflated all the time. The system uses portable compressor that will supply air to all four tires via hoses and a rotary joint fixed between the wheel spindle and wheel hub at each wheel. The rotary joints effectively allow air to be delivered to the required under inflated tire. This system acknowledges a essential improvement in gas mileage, tire wear reduction, and an increase in handling and tire performance in diverse conditions.

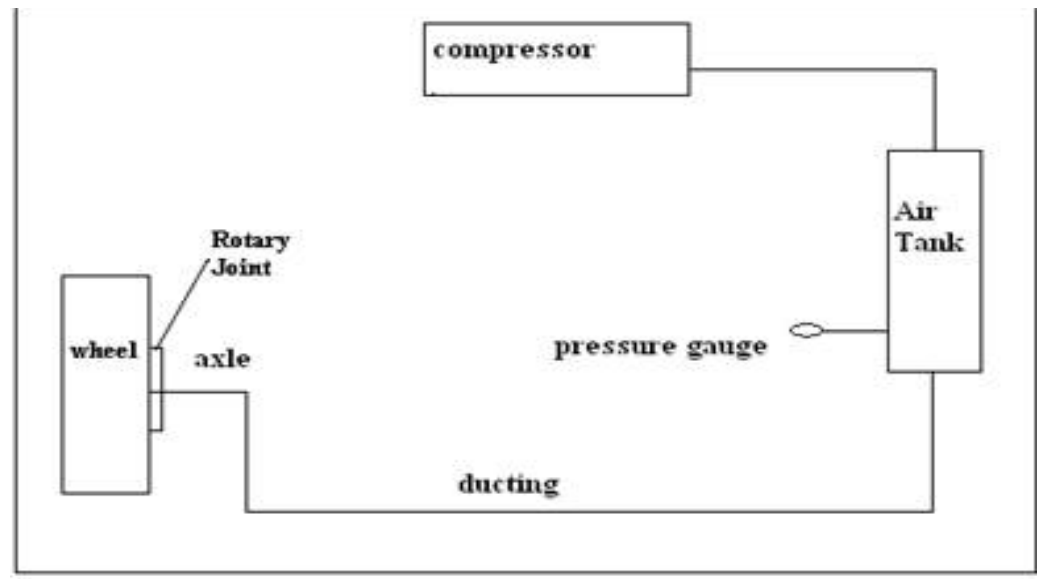

Fig. 2 Block Diagram of air inflating system.

S. Adakmol et al [2]. focused attention to the reasons due to which tires lose air and thus tried to develop the automatic system so that vehicle run safely on road all the time without attaining the under inflating condition ever hence avoiding the accidents. Centralized compressor system is applied over in this paper system. Under inflated tires overheat very rapidly then a well inflated tire which damages the tires within very short time span. In this system as soon as tire pressure goes under inflated state then a pressure sensor senses it and send it to all the controller which activates the solenoid valve and air is filled up to the exact pressure. Hemantsoni et al. [6] has studied for automatic tire inflation system and he introduced the centralized compressor based system with the aim of improving gas mileage, tire life, car safety and working. Using this system air is delivered to all four tires of the vehicle using hoses and a rotary joint constrained between the wheel spindle and wheel hub at every wheel. This is most beneficial project for society as the system automatically refills all the four tires whenever required under all environmental and road conditions.

P. Omprakash et al. [7] worked on the mechanism for air refilling system. The aim of this project was to introduce a system that can be used in any type of tire either tube or tubeless. The system uses the permanent connection between valve and hoses with only intention of providing tight connection during rotation of tire so that it can be lose only when substituting the tire by the driver. Other intention expressed through this paper is that the vehicle tire must never be either under inflated nor over inflated. Pressure in tires should be always idealized level as under inflation leads to wearing of tire, consumption of excess fuel and over inflation causes explosion of tires. This system also help predict about the puncture in the tire when there is continuous reduction in pressure of its set threshold value as shown in the Fig. $(3,4)$.

H. Soni et al. [8] investigated the result of drop in tire pressure. Driven by their studies developed a compressor

to obtain air from atmosphere, compress it and supply it to the tire for pressure regulation. The system automatically examines the tire pressure using pressure gauge. If the tire is under inflated then the compressor 
starts to deliver air to inflate the tire. The switching of the circuit takes place using electronic circuits. This system has taken into consideration all the growing environmental issues and has addressed the system to work in all diverse conditions

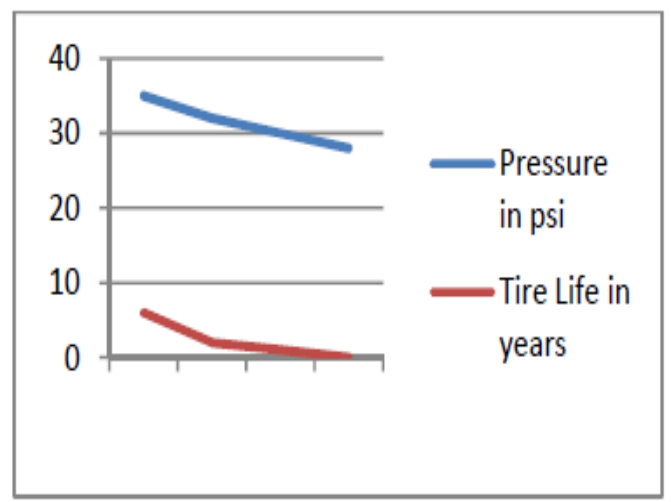

Fig. 3 Before system graph

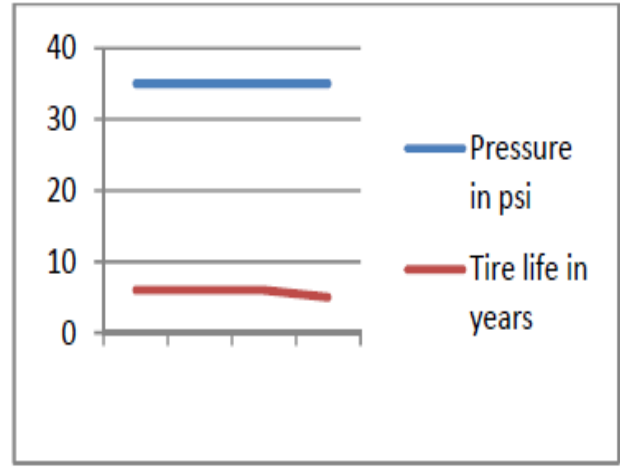

Fig. 4 After system graph

O.H. Latha et al. [9] worked on solar based air compressor for inflating tires. Sun is renewable source of energy and radiations received from Sun are received by the Solar Photovoltaic Cell, the energy is kept stored in battery back. From battery back it is supplied to the air compressor if it is DC motor. The voltage regulator, microcontroller are used to regulate the variations in the voltage. From batteries the energy is changed for AC motors air compressors. As the battery is charged then energy is supplied to air compressor and it starts working as shown in Fig. 5.

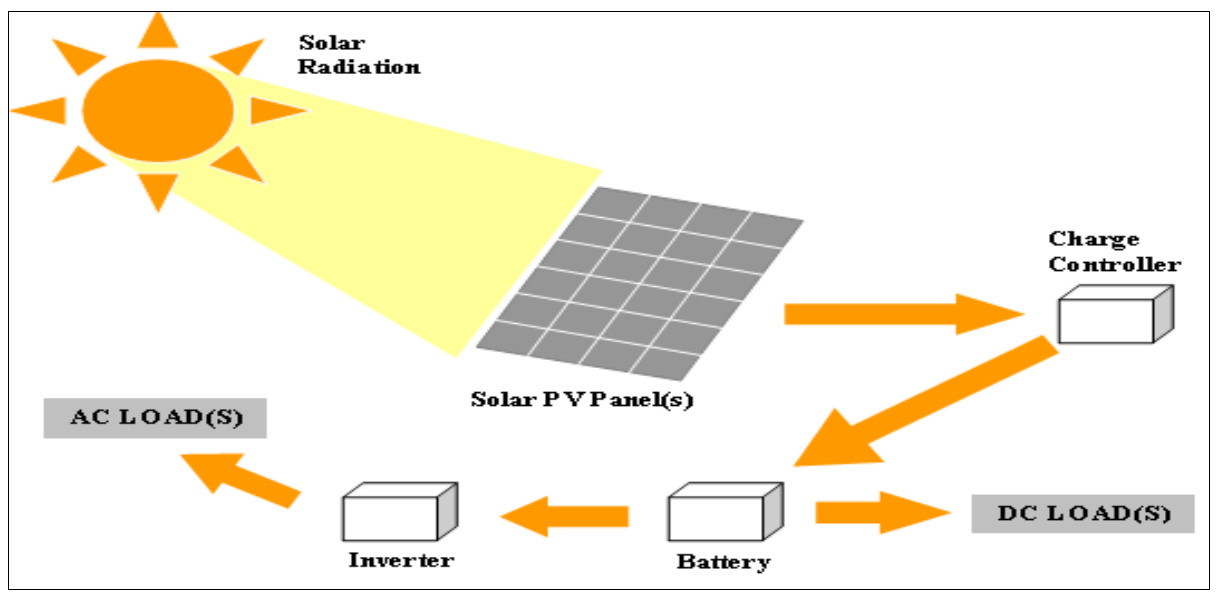

Fig. 5 Diagram of Solar based air compressor for inflating tires.

L. chandreshkumar et al. [11] examined inappropriate tire pressure and temperature related issues in automobiles. Tire monitoring system is introduced in this paper to overcome all tire pressure related problems is being classified as Direct and Indirect system. In Direct system sensors, receivers and transmitters are used. Sensors checks for reduction in tire pressure and transmits the alert signal. While in Indirect TPMS tire pressure alerting signal is not involved. The software is fixed on the wheel which checks the reduction in pressure on the basis of wheel speed. When the tire pressure drops, the automobile weight makes the tire to rotate at a speed varied from the one which is obtained when it is at appropriate pressure. Hence only software is required to implant an indirect TPMS.

M. A. Ajas et al. [4] investigated tire pressure monitoring and automatic pressure filling system for commercial vehicles. It is an electronic system designed to monitor air pressure and temperature in the tire and user is alerted by providing the result display. If the pressure is below the required level then the compressor will inflate the tire. If it is above expected pressure then excess amount will be released through the valve. TPMAFS maintains the standard pressure and helps avoid accidents occurring due to low pressure tires.[10] 
A. Mathai et al., worked on tire pressure monitoring system and provided a new approach to inflate the low pressure tires. In this system current pressure in the tire and low or excess pressure in the tire is displayed combined with LED lights for providing users with alert warning. The system works on wireless sensor network using Zigbee and CAN Communication protocol. The data received wirelessly through Zigbee will be converted to CAN protocol format.

Sivarao et al. [13] investigated a method to ease the users to refill the under inflated tires. By the technique introduced in this paper the driver no more needs to remember the tire pressure value. Once the exact required value is reached then the device makes hissing sound to make user aware of change in pressure value. This device can also be used together with industrial air compressor or any other air pressure resources. No counter checking required, multilevel pre-set can be achieved, and most importantly can be handled by anybody including kids above 6 years.

M. Reiter et al. [12], worked on an automated automobile tire inflation system and put forth the consequences of the varying tire pressure on automobile handling. In this research paper a numerical investigation has been given for various tire inflation pressures using STI tire model. Results have been revealed using analysis on longitudinal and lateral forces and plus aligning torque. A quadruple lane test clearly came up with result that the required steering wheel angle increases up to $47.7 \%$ for front axle tire inflation pressure at $70 \%$ of normal values, where the vehicle slip angle was up to $77.8 \%$ larger when all tires were inflated to $70 \%$ of recommended pressure. All these analysis concluded that the under inflated front axle tires result in under steer tendencies

while rear axle under inflation creates over steer behavior.

K. Vishnuram. et al. [3] examined self-inflating tire system and came up with the design to constantly maintain tire pressure at the appropriate level. There are many of self- inflating tire systems in the market, but lots of them are only mean for the military and commercial application automobiles only. Hence this research paper introduced this system with the goal to constantly monitor the air pressure in each and every tire, and if any problem is detected in the tire than to immediately alert the user and to keep check on air supply as well as examine the check valve so that it should open only when needed has been achieved.

\section{CONCLUSION}

- Automatic pressure controlling and self- inflating system would be definitely exploding as new product in the automobile supplier industry as such a product does not currently installed for majority of passenger automobiles, hence the market conditions would be favorable to release such a system.

- It specially satisfies the user requirement by maintaining ideal tire pressure for under inflated tires, also improves fuel efficiency and overall safety of automobile is concerned.

- It assists in monitoring appropriate tire pressure constantly, reduces or increases the tire pressure according to requirement of the tire and help in gaining best mileage and most importantly assures to provide comfortable and safe driving.

- The installation of such a system in vehicles is a low cost affair so all the passenger vehicle can take the advantage of this very essential system at affordable installation budget.

\section{REFERENCES}

[1] I. Burase, S. Kamble, A. Patil and A. Kharat." A Survey on Automatic Air Inflating System for Automobile". International Journal of Innovative Research in Science, Engineering and Technology, Vol. 5(10), 2016, ISSN: 23198753

[2] G. Ramesh, M. Mallesha and V.Gangadhar." Effect of Tyre Overload and Inflation Pressure on Rolling Loss in Cars" Int. Journal of Engineering Research and Applications, , Vol. 5(8),2015, pp.01-08, ISSN: 2248-9622

[3] S. Adakmol, T. Shende, D. Poriya, S. Fotedar and S.P.Shinde."Central Tyre Air Inflation System" IJSRD International Journal for Scientific Research \& Development| Vol. 4(3), 2016, ISSN:2321-0613.

[4] K. Vishnuram, R. Dinesh, S. Krishna, D. Kumar and T. Chazhiyan.” Self Inflating Tyres” International Journal on Applications in Mechanical and Production Engineering, Vol.1(5),2015, pp 5-6, ISSN :2395-3500

[5] A. Mathai and P. Ranjan." A New Approach to Tyre Pressure Monitoring System" International Journal of Advanced Research in Electrical, Electronics and Instrumentation Engineering. Vol. 4(2), 2015.

[6] H. Soni, A. Lahurgade, S. Relkar and S. ,Babhulkar.” Automatic Tyre Inflation System,Vol. 3(10), 2014. ISSN 2231-5063.

[7] P. Omprakash and T. Kumar. "M.A.R.S - Mechanized Air Refilling System", International Journal of Information Sciences and Techniques, Vol.4(3), 2014.

[8] H. Soni, P. Golar and A. Kherde, "Design of Automatic Tyre Inflation System”. Industrial Science, Vol.1(4), 2014. ISSN : 2347-5420.

[9] O. H. Latha1, S.I. Sadaq and M. A. R. Junaidi, " Solar Based Air Compressor for Inflating Tyres". IOSR Journal of Mechanical and Civil Engineering (IOSR-JMCE), Vol. 11(5),2014, pp. 29-33, ISSN: 2278-1684 
[10] Ajas.M.A, Aiswarya.T.G, Adersh Vinayak, Surya Balakrishnan and P.S Janahanlal." Tire Pressure Monitoring and Automatic Air Filling System" IJREAT International Journal of Research in Engineering \& Advanced Technology, Vol.2( 2),2014, ISSN: $2320-8791$.

[11] L. Chandreshkumar, J. Pranav, ,C. Hemra and Bokade ." Tire Pressure Monitoring System And Fuel Leak Detection" International Journal of Engineering Research and Applications (IJERA),Vol. 3(3), 2013, pp.345-34, ISSN: 22489622

[12] M. Reiter and J. Wagner, P.E Automated Automotive Tire Inflation System -Effect of Tire Pressure on Vehicle Handling. 6th IFAC Symposium Advances in Automotive Control Munich, 2010,pp.12-14

[13] Sivarao, T.J.S. Anand and M.Warikh.” Engineering of Tire Pressure Controlling Device:An Invention Towards Successful Product Development" 